\title{
Impacto de la caries dental en la calidad de vida en niños de 11 a 12 años de la Unidad Educativa Dolores J Torres, Parroquia Sagrario, Cuenca
}

\author{
Impact of dental caries on the quality of life in children of 11 to 12 years of the Dolores $\mathrm{J}$ Torres \\ Educational Unit, Sagrario Parish, Cuenca.
}

Zhunio Ordoñez Karla ${ }^{1 \mathrm{a}}$,Urgiles Cristian ${ }^{1 \mathrm{~b}}$

\begin{abstract}
RESUMEN
Objetivo: El objetivo de este trabajo fue establecer el impacto de la caries dental y la prevalencia de las condiciones bucales sobre los desempeños diarios en la calidad de vida en escolares de 11 y 12 años de edad de la Unidad Educativa Dolores J. Torres, parroquia Sagrario, Cuenca, 2015. Materiales y Métodos: Este estudio es de tipo descriptivo, observacional, de corte transversal. El universo fue de 115 escolares, de los cuales se tomó una muestra de 90 participantes con una media de edad de $11.24 \pm 0.42$ (DT), el 100\% fueron del sexo femenino, Los datos fueron recolectados a través de una entrevista, empleando el instrumento OIDP versión en español. Resultados. El 100\% de las niñas manifestaron uno o más de un desempeño impactado. El problema oral que más afecta es el mal aliento seguido de los dientes sensibles y los dientes cariados, los mismos que han sido molestos al momento de comer o higienizarse la boca. $73,3 \%$ de las escolares presenta de 1 a 4 obturaciones. El $36,7 \%$ de las niñas están libres de caries dental. Una de las 90 participantes ha perdido una pieza dental permanente. La diferencia de las niñas que tuvieron o no caries solo varia al momento de descansar y en las labores escolares. Conclusiones: La prevalencia del impacto de las condiciones orales sobre la CVRSB de las escolares fue muy alta (100\%), lo cual significa que todas las escolares mostraron al menos un desempeño impactado, siendo los más altos: comer y sonreír.
\end{abstract}

PALABRAS CLAVE: Calidad de vida; Salud Bucal; Desarrollo Infantil; Caries Dental.

\section{ABSTRACT}

Objective: The objective of this work was to establish the impact of dental caries and the prevalence of oral conditions on daily performance in the quality of life in 11 and 12 year old schoolchildren of the Dolores J. Torres Educational Unit, Sagrario Parish, Cuenca, 2015.Materials and Methods: This study is descriptive, observational, cross-sectional. The universe was 115 schoolchildren, of which a sample of 90 participants with an average age of $11.24 \pm 0.42$ (DT) was taken, $100 \%$ were female, the data were collected through an interview, using the OIDP instrument Spanish version. Results. $100 \%$ of the girls showed one or more of an impacted performance. The oral problem that most affects is bad breath followed by sensitive teeth and decayed teeth, which have been annoying at the time of eating or cleaning the mouth. $73.3 \%$ of schoolchildren have 1 to 4 seals. $36.7 \%$ of girls are free of tooth decay. One of the 90 participants has lost a permanent dental piece. The difference of girls who had or did not decay only varies at the time of rest and in school work. Conclusions: The prevalence of the impact of oral conditions on the HRQL of schoolchildren was very high (100\%), which means that all schoolchildren showed at least an impacted performance, the highest being: eating and smiling.

KEY WORDS: Quality of Life; Oral Health; Child Development; Dental Caries.

${ }^{1}$ Universidad Católica de Cuenca. Ecuador

a Odontóloga pasante de investigación en Odontología b Odontólogo Especialista en Rehabilitación Oral, docente

\section{Correspondencia:}

Karla Zhunio

Correo electrónico: kzhunio93@gmail.com
Este es un artículo Open Access distribuido bajo la licencia Creative Commons Atribución--No Comercial-.-Compartir Igual 4.0

Citar como: Zhunio K, Urgiles C. Impacto de la caries dental en la calidad de vida en niños de 11 a 12 años de la Unidad Educativa Dolores J Torres, Parroquia Sagrario, Cuenca. KIRU. 2020;17(2): 69- 74. https://doi.org/10.24265/kiru.2020.v17n2.02 


\section{INTRODUCCIÓN.}

De acuerdo a la Organización Mundial de la Salud (OMS) el $60 \%-90 \%$ de los escolares y casi el $100 \%$ de las personas adultas poseen caries dental a nivel mundial, dando énfasis en los factores de riesgo tales como: hábitos malos de alimentación, el tabaquismo, el consumo excesivo de alcohol y escasa higiene bucodental, además de varios determinantes sociales $^{(1)}$.

"Las enfermedades bucodentales pueden convertirse en un obstáculo importante para disfrutar de una vida saludable", afirma Saskia Estupiñán, experta en Salud Bucodental de la Organización Panamericana de la Salud/Organización Mundial de la Salud (OPS/OMS) (2).

El objetivo de del presente es determinar cómo impactan las Condiciones orales sobre la Calidad de Vida Relacionada a la Salud (CVRS) en el grupo de escolares de 11 y 12 años de la Unidad Educativa "Dolores J. Torres", parroquia El Sagrario, Cuenca.

Para evaluar esta patología se empleó el índice CPO$D$ en dientes permanentes y el índice ceo-d en dentición primaria ${ }^{(30}$.

El cuestionario Child OIDP es un instrumento validado y estandarizado, el mismo que nos permite determinar el impacto en la calidad de vida relacionada con la salud por factores bucales concretos de acuerdo a la autopercepción del encuestado ${ }^{(4)}$.

\section{MATERIALES Y MÈTODOS}

El estudio se efectuó en escolares de 11 a 12 años de edad, que pertenecían al séptimo año de Educación General Básica de la Unidad Educativa. Este fue un estudio: observacional, transversal, cuantitativo a nivel descriptivo con alcance correlacional. Se consiguió el asentimiento del Comité Institucional de Ética de la Universidad Católica de Cuenca-Ecuador, la autorización de la Directora de la Institución Educativa, el consentimiento informado de los padres de familia y el asentimiento de las niñas.

La población fue de 115 estudiantes, y la muestra de 90 participantes con una media de edad de $11.24+$

0.42 (DT), el $100 \%$ fueron del sexo femenino, que estuvieron matriculadas el año 2015 en la Unidad Educativa "Dolores J. Torres", de la parroquia urbana El Sagrario de Cuenca-Ecuador. Se tomaron como criterios de inclusión los siguientes: escolares de 11 y 12 años de edad cumplidos, aparentemente sanas, de sexo femenino, con autorización de la institución educativa, con Consentimiento Informado de los padres y Asentimiento Informado de las niñas. Los criterios de exclusión fueron: estudiantes fuera del rango de edad, escolares que no contaban con el asentimiento o consentimiento informado por parte de su representante. Se utilizó el método de observación y encuesta empleando el cuestionario validado Child OIDP versión en español ${ }^{(5)}$.

En primera instancia las escolares tenían que señalar los ítems de una lista de condiciones bucales, los que la estudiante sentía como un problema en su vida cotidiana. Luego en otra parte se evaluó la severidad, intensidad y extensión del impacto ${ }^{(6)}$. Anteriormente se calculó qué desempeños habían sido afectados por las condiciones bucales, indagando a la encuestada lo siguiente: "en los últimos tres meses, ¿alguno de estos problemas con tu boca o dientes te ha dado problemas para... (desempeño) ${ }^{(7)}$. Si la respuesta era negativa, entonces se consideraba en los casilleros de severidad y frecuencia el valor de cero. Si la respuesta era positiva, se indagaba por la severidad y frecuencia de cada desempeño ${ }^{(8)}$. Para establecer la severidad, se le indicó a cada niña una flecha horizontal de manera creciente en la cual se podía ver los tres valores de severidad (poco, regular, mucho) ${ }^{(9)}$. Para lo cual, se preguntó lo siguiente:

¿cuánto ha afectado tu vida diaria la dificultad para... (desempeño)? Para determinar la intensidad se valoró la frecuencia, en la escala de 1 a 3 , se indagó a cada escolar cuántas veces se presentó la dificultad en los últimos tres meses, la consulta que se les realizó fue: ¿la dificultad para... (desempeño) se te ha presentado: una o dos veces en un mes? (según la escala corresponde al valor 1), ¿tres a más veces en un mes? (según la escala corresponde al valor 2) o ¿más de tres veces en los últimos tres meses? (según la escala corresponde al valor 3$)^{(10)}$. Para determinar la extensión: la misma que hace referencia al número de desempeños impactados que afectan la vida cotidiana del estudiante. Se le preguntó a las escolares: de acuerdo a los problemas de tu boca o dientes ¿puedes especificar cuál de ellos es (son) la(s) causa(s) de la dificultad para... (desempeño)? La extensión podía ir desde el valor 0 (que significa ningún desempeño impactado) hasta el valor 8 (todos los desempeños impactados). El procesamiento y análisis de los datos se ejecutó con el programa Excel $2010^{(11)}$.

\section{RESULTADOS}

Al hablar de Incidencia de los impactos bucales en los últimos tres meses las niñas consultadas indicaron que el problema bucal que más les molesta es el mal aliento $(70 \%)$, seguido de los dientes sensibles $(68,9 \%)$ y los dientes cariados $(61,1 \%)$, Entre los problemas menos frecuentes están: la pérdida de dientes definitivos $(1,1 \%)$, las fracturas de dientes $(1,1 \%)$ y las llagas en la boca $(3,3 \%)$. La única condición que no fue reportada constituye la deformación de la boca o cara. (Tabla1) 
Tabla 1. Prevalencia de las condiciones orales percibidas en escolares de 11 y 12 años de la Unidad Educativa "Dolores J. Torres" Cuenca - Ecuador.

\begin{tabular}{lcccc}
\hline \multirow{2}{*}{ Condiciones bucales } & \multicolumn{3}{c}{ Si } & No \\
\cline { 2 - 5 } & $\mathbf{n}$ & $\%$ & $\mathbf{N}$ & $\%$ \\
\hline Dolor de muela & 47 & 52.2 & 43 & 47.8 \\
Diente sensible & 62 & 68.9 & 28 & 31.1 \\
Diente cariado & 55 & 61.1 & 35 & 38.9 \\
Caída diente leche & 52 & 57.8 & 38 & 42.2 \\
Dientes separados & 15 & 16.7 & 75 & 83.3 \\
Fractura de dientes & 1 & 1.1 & 89 & 98.9 \\
Color & 45 & 50 & 45 & 50 \\
Forma & 45 & 50 & 45 & 50 \\
Posición & 42 & 46.7 & 48 & 53.3 \\
Sangrado & 42 & 46.7 & 48 & 53.3 \\
Inflamación & 29 & 32.2 & 61 & 67.8 \\
Sarro & 11 & 12.2 & 79 & 87.8 \\
Llagas & 3 & 3.3 & 87 & 96.7 \\
Mal aliento & 63 & 70 & 27 & 30 \\
Deformación & 0 & 0 & 0 & 0 \\
Erupción & 18 & 20 & 72 & 80 \\
Pérdida de dientes permanentes & 1 & 1.1 & 89 & 98.9 \\
\hline
\end{tabular}

El 96.7\% de las escolares indican que, en los últimos tres meses, los problemas orales les han afectado al momento de: comer alimentos o limpiarse la boca, el $90 \%$ declara que le afectan al sonreír. El desempeño con menor grado de afectación es el de realizar labores escolares con el 68,9\%, seguido del contactarse con otros individuos, con el $77,89 \%$. (Gráfico 1- )

Tabla 2. Intensidad de los impactos percibidas en escolares de 11 y 12 años de la Unidad Educativa "Dolores J. Torres" Cuenca - Ecuador.

\begin{tabular}{|c|c|c|c|c|c|c|c|c|}
\hline Nivel impacto & Comer & $\begin{array}{l}\text { Hablar } \\
\text { claramente }\end{array}$ & $\begin{array}{l}\text { Limpiarse } \\
\text { la boca }\end{array}$ & Descansar & $\begin{array}{l}\text { Buen } \\
\text { ánimo }\end{array}$ & Sonreír & $\begin{array}{l}\text { Labores } \\
\text { escolares }\end{array}$ & $\begin{array}{l}\text { Relacionars } \\
\text { e }\end{array}$ \\
\hline & $\%$ & $\%$ & $\%$ & $\%$ & $\%$ & $\%$ & $\%$ & $\%$ \\
\hline -Muy poco & 10.0 & 37.8 & 18.9 & 34.4 & 48.9 & 14.4 & 44.4 & 38.9 \\
\hline -Poco & 17.8 & 18.9 & 36.7 & 11.1 & 22.2 & 8.9 & 13.3 & 23.3 \\
\hline -Moderado & 27.7 & 20.0 & 18.9 & 13.3 & 7.8 & 32.2 & 10.0 & 10.0 \\
\hline -Severo & 33.3 & 1.1 & 14.4 & 14.4 & 2.2 & 28.9 & 1.1 & 5.6 \\
\hline -Muy severo & 8.9 & 1.1 & 7.8 & 5.6 & 0.0 & 5.6 & 0.0 & 0.0 \\
\hline
\end{tabular}




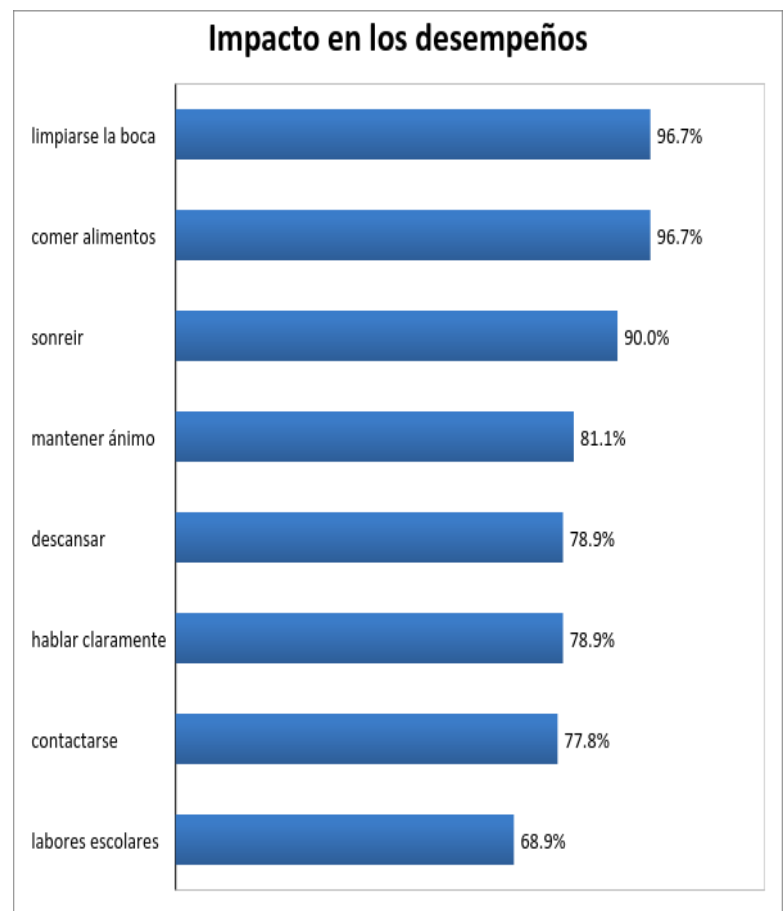

Gráfico 1. Impacto de la salud oral en los desempeños en escolares de 11 y 12 años de la Escuela Fiscal "Dolores J. Torres" Cuenca - Ecuador.

Como se aprecia en la (tabla 2) los desempeños afectados con mayor grado de frecuencia son: comer y sonreír, un 33,3\% de escolares manifiestan haber sido afectadas en un nivel severo en el instante de comer los alimentos, un $32,2 \%$ de estudiantes indican que fueron afectadas moderadamente al: sonreír, reír o mostrar los dientes. Los desempeños de menor frecuencia son: el buen ánimo, seguido de la elaboración de labores escolares; el $48.9 \%$ y el $44,4 \%$ de escolares señalan que se vieron afectados MUY POCO en cada uno de dichos desempeños.

De las 90 escolares, 54 especifican como poca la severidad de los impactos en su desempeño, 25 lo señalan como regular y 11 como severo

De las 90 participantes, 54 indican poca la severidad de los impactos en su desempeño, 25 lo determinan como regular y 11 como severo. De las 90 niñas, 75 escolares indican que la dificultad en los desempeños en los últimos tres meses se ha presentado una o dos veces al mes (leve), 14 estudiantes señalan que se han visto afectadas dos o tres veces al mes (moderado) y una sola estudiante manifiesta que ha presentado problemas más de tres veces al mes (severo). (Gráfico 2 y 3).

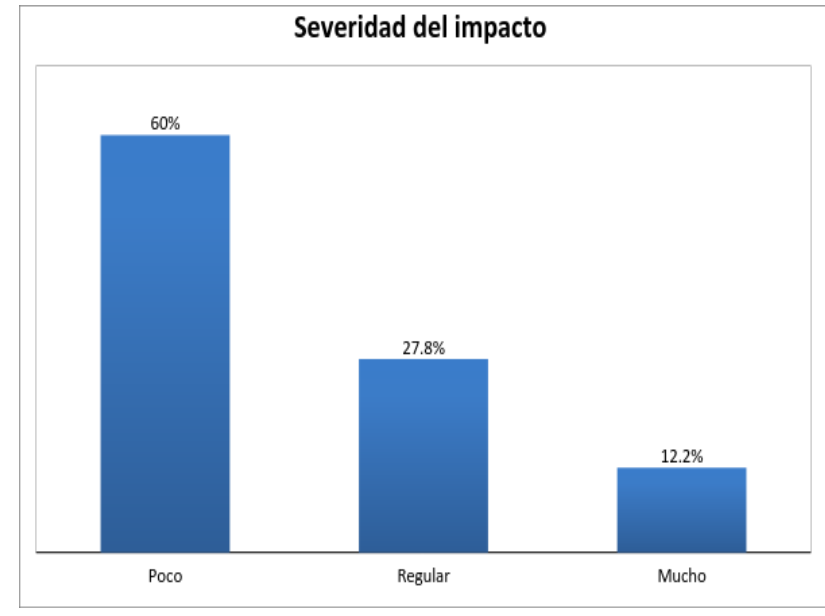

Gráfico 2. Nivel de severidad de los desempeños en escolares de 11 y 12 años de la Unidad Educativa "Dolores J. Torres" Cuenca - Ecuador.

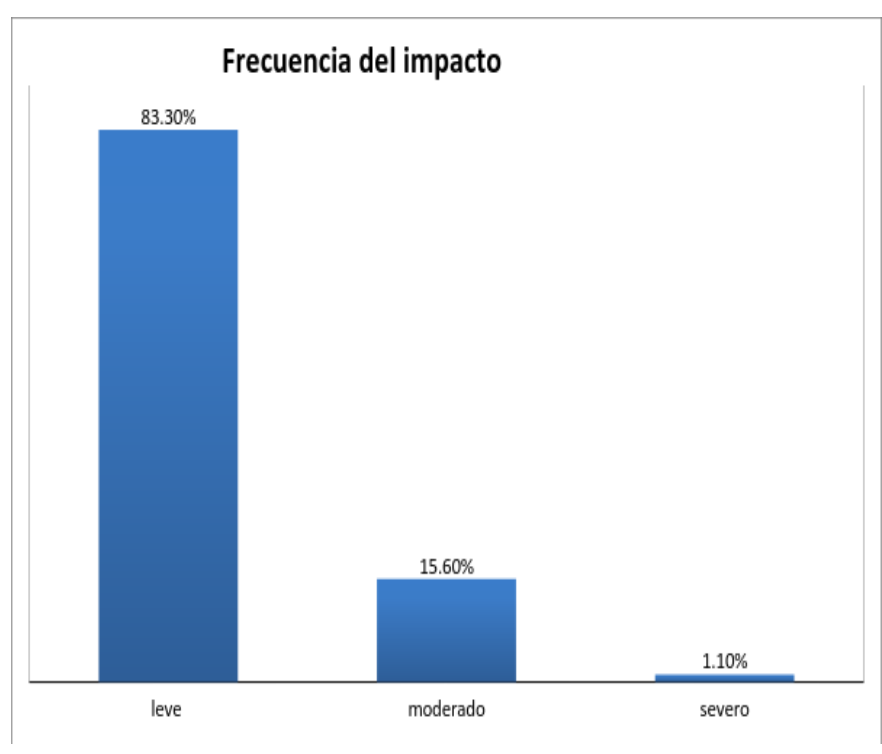

Gráfico 3. Frecuencia del impacto en los desempeños en escolares de 11 y 12 años de la Unidad Educativa "Dolores J. Torres" Cuenca - Ecuador.

Se comprobó que existe una correlación estadísticamente reveladora en tener caries dental y la percepción de impacto en los desempeños relacionados con descansar y realizar labores escolares. (Tabla 3) 
Tabla 3. Asociación entre tener caries y el impacto en los desempeños en escolares de 11 y 12 años de la Unidad Educativa "Dolores J. Torres" Cuenca - Ecuador.

\begin{tabular}{lcccccc}
\hline & \multicolumn{2}{c}{ Sin caries } & \multicolumn{2}{c}{ Con caries } & \multicolumn{2}{c}{ Estadísticos } \\
\cline { 2 - 7 } veseringerius & $\mathrm{n}$ & $\%$ & $\mathrm{n}$ & $\%$ & $\mathrm{X}$ & $\mathrm{P}$ \\
\hline Descansar & 21 & 23.3 & 50 & 55.6 & 7.278 & $0.007^{*}$ \\
Estudios & 13 & 14.4 & 49 & 54.4 & 21.15 & $0.000^{*}$ \\
& & & & & & \\
\hline
\end{tabular}

\section{DISCUSIÓN}

Mediante los resultados que se obtuvieron en este trabajo se pudo comprobar que hay una alta prevalencia del impacto de las condiciones bucales sobre la CVRSB de los escolares debido a que el $100 \%$ de las niñas examinadas declaró al menos tres impactos en alguno de sus desempeños diarios en los tres meses anteriores al estudio.

Los resultados de este trabajo muestran que los impactos bucales que más les molesta a las niñas son el mal aliento (70\%) y los dientes sensibles (68.9\%) y los menos frecuentes son fracturas de dientes (1.1\%) y llagas en la boca /3.3\%). Según César del CastilloLópez y col. en Perú $2014^{(12-13)}$. Los impactos orales con mayor afectación son el dolor de muela $(58,0 \%)$ y diente sensible (32,7\%). Los de menor impacto fueron: fractura de diente permanente y sangrado de encías ( $1,3 \%$ en cada caso) datos que tienen similitud con los encontrados por Evelyn Roxana Paredes-Martínez, María Elena Díaz-Pizán, Lima Perú $(2014)^{(14-15)}$ que de los 169

estudiantes examinados el $60.9 \%$ (103) manifestaron la condición bucal: dolor de muela como primer problema, el 51,5\% (87 estudiantes) percibieron como problema el diente sensible.

En este trabajo los desempeños más afectados son al comer y al limpiarse la boca (96.7\%) y al sonreír $(90 \%)$ y los menos señalados son el realizar las labores escolares (68.9\%) y contactarse con otras personas con el (77.89\%). Estos valores poseen semejanzas con los manifestados por YUSUF et, al, ${ }^{(16-}$

17) en su revisión de 8 artículos relata el comer (23.2\%) y limpiarse los dientes (18.0\%) son los desempeños más afectados y los menos afectados son realizar las labores escolares (1.8\%) y contactarse $(2.2 \%)$ al igual que tiene cierta semejanza con los datos obtenidos por Del Castillo et al. ${ }^{(18-19)}$ en cuyo estudio los desempeños más impactados fueron comer, limpiarse la boca y emoción y los menos impactados fueron: estudiar, dormir y contactarse. En cambio, que para Paredes y col. Los más afectados fueron comer y sonreír y los menos afectados estudiar y emoción ${ }^{(20)}$.
La prevalencia de impactos descubierta en los estudiantes de a zona Urbana de Cuenca fue elevada en comparación con la examinada en la zona rural en Piura y urbano de Zapallal, Lima ${ }^{(21)}$.

Las condiciones bucales que impactan en la CVRSB son diferentes en una zona y otra y entre países se nota más aun las diferencias, esto es por la mezcla de razas las condiciones climáticas y las mismas condiciones sociales de cada país. A pesar de ello el índice Child-OIDP, que evalúa el impacto de las condiciones bucales en la CVRSB, tuvo un puntaje promedio global de 27.13 tanto que el impacto global en los 150 escolares examinados de Piura obtuvo un puntaje de 7,05. Este promedio es similar a lo manifestado en estudiantes de diferentes sitios poblacionales: 7.7 en Lima (Zapallal), estas discrepancias corresponderían a las diferentes características socio-geográficas.

Sin embargo, solo una estudiante señaló tener la ausencia de una pieza dentaria permanente similar a los estudios ejecutados en Perú que asimismo fue minima la incidencia en dientes permanentes perdidos, esto es expiado en diferentes estudios que han demostrado que a esa edad es mínima la usencia de dientes definitivos faltantes.

Se concluye que la prevalencia del impacto de las condiciones bucales sobre la CVRSB de las niñas de 11 a 12 años de la Unidad Educativa "Dolores J. Torres" Cuenca-Ecuador, fue elevada (100\%), lo cual indica que todas las 90 estudiantes evaluadas mostraron al menos un desempeño diario impactado, siendo los más afectados: comer y sonreír.

Contribuciones de autoría: $\mathrm{KZ}$ y $\mathrm{CU}$ diseñaron el estudio, recopilaron y analizaron los datos, redactaron y aprobaron el manuscrito.

Fuente de financiamiento: Autofinanciado.

Conflicto de intereses: Los autores declararon no tener conflictos de interés. 


\section{REFERENCIAS BIBLIOGRÁFICAS}

1. Organización Mundial de la Salud. (Abril de 2012). Salud Bucodental. Nota informativa .Centro de prensa. (N॰318.).

2. Organización Panamericana de Salud. (2013.). La salud bucodental es esencial para la salud general. OPS., México.

3. Moreira, D. ( Cuba 2011.). La caries Dental. Revisión Bibliográfica. . Portal de Evelio E. Moreira Díaz.

4. Chavez, J. M. (Enero de 2012). ncidencia del proceso carioso en los primeros molares permanentes en pacientes de 7 a 10 años que acuden a la clínica de odontopediatria en el mes de enero del 2012 . Universidad De Guayaquil .

5. Klein, H., C. P., \& Knutso., a. J. (s.f.). "Studies on Dental Caries: Dental Status and Dental Needs of Elementary School Children. Public Health Reporter, 53 (1938,), págs. 751-765.

6. Erik., P. P. (Argentina 2003). Continuous improvement of oral health in the 21st century. The approach of the WHO Global Oral Health Programme.

7. Roxana., P. M. (Perú 2013). Lima Impacto de las condiciones orales sobre la calidad de vida en escolares del distrito de villa el salvador en el año 2013. . Herediana .

8. Tsakos, G. (2006). Validation of an English version of the Child-OIDP index, an oral health-related quality of life measure for children,. Departamento de Odontología Comunitaria de la Facultad de Odontología de la Universidad de Chulalongkorn. Bangkok, T .

9. Meriel, E., M, J., \& Stephen, C. (Brasil2010). Measuring quality of life. .

10. Ximena, R., \& Constanza, V. (2013). Impacto de la salud bucal en la calidad de vida de escolares de 11 a 14 años. . Licantén, ELSEIVERDOMA .

11. César del Castillo, L., Alexis, A., \& Pablo, B. (Piura, Peru. 2014). Impact of oral conditions on the quality of life in rural schoolchildren,. Herediana .

12. Evelyn, P. M., \& María Elena, D. (2014). Impacto de las condiciones bucales sobre la calidad de vida en escolares del Distrito de San Juan de Miraflores. Lima, Revista Estomatológica Herediana, 24 (3).
13. Natalia Koposova, W., Martin, E., Roman, K., \& Harald, E. (Rusia 2010). Oral health and quality of life in Norwegian and Russian school children: A pilot study Stomatologija, Baltic Dental and Maxillofacial Journal,. Dentistry Journals .

14. Janice, P., Marcelo, C., Meneghim, P., \& Fábio, L. ( 2015.). Oral health, socio-economic and home environmental factors associated with general and oral-health related quality of life and convergent validity of two instruments. BMC Oral Health , 13:10;

15. Huda, Y., Sudaduang, G., Aubrey, S., Georgio, \& Tsakos. (2006). Validation of an English version of the Child-OIDP index, an oral. Health Qual Life Outcomes. , 4: 38.

16. Nazik, N., Mutaz, A., Tordis, T., \& Anne, Å. ( 2010; ). Evaluation of oral health-related quality of life among Sudanese schoolchildren using ChildOIDP inventory. Health Qual Life Outcomes., 8: 152.

17. World Health Organization; . (1997.). The World Health Organization quality of life instruments. Geneva: . World Health Organization. WHOQOL measuring quality of life.

18. Alvarez, J., Bönecker, M., Prócida, D., \& Raggio, D. ( Brasil 2010). .Impacto de los problemas bucales sobre la calidad de vida de niños. . Rev Estomatol Herediana..

19. Candiales de Castillo, Y., Cabello, I. R., Urzúa, A. I., \& Rodríguez Martínez, G. (Santiago 2013). Aspectos sobre la medición del impacto de la caries dental en la calidad de vida de las personas: Artículo de revisión. Revista clínica de periodoncia, implantología y rehabilitación oral. , vol.6 (no.1).

20. M., A. U. (Santiago mar. 2010). Calidad de vida relacionada con la salud: Elementos conceptuales. Rev. méd. Chile ., v.138 (n.3 ).

21. Tapias, L., \& fortich, T. (2013). Percepción de calidad de vida relacionada con la salud oral en los pacientes adultos atendidos en la clínica de odontológica de la corporación universitaria Rafael Núñez en Cartagena de Indias periodo del año 2013. corporación universitaria Rafael Núñez en Cartagena de Indias, Cartagena.
Karla Zhunio

kzhunio93@gmail.com

Christian Urgiles

curgilesu@ucacue.edu.ec
0000-0002-7430-0917

0000-0003-4077-4601 